\title{
A novel combination of bioresorbable polymeric film and expanded polytetrafluoroethylene provides a protective barrier and reduces adhesions
}

\author{
Sunjay Kaushal, MD, PhD, ${ }^{\text {a,b }}$ Shivani K. Patel, BS, ${ }^{\text {a }}$ Saik-Kia Goh, BS, a Aditya Sood, BS, ${ }^{\mathrm{a}}$ \\ Brandon L. Walker, MSc, ${ }^{a}$ and Carl L. Backer, MD ${ }^{\text {a,b }}$
}

\begin{abstract}
Objective: A bioresorbable polymeric film reduces the extent and severity of postoperative adhesions in infants undergoing repeat sternotomy. Resorption of the bioresorbable polymeric film, however, leaves no barrier between the sternum and the epicardium. A sheet of expanded polytetrafluoroethylene is used by many surgeons to create a physical barrier between the sternum and the cardiac structures. We hypothesized that placing bioresorbable polymeric film beneath an expanded polytetrafluoroethylene pericardial membrane would both decrease pericardial adhesions and provide a physical barrier.
\end{abstract}

\begin{abstract}
Methods: A novel combination of bioresorbable polymeric film underneath an expanded polytetrafluoroethylene membrane was tested in an established rabbit model of pericardial adhesion formation. After sternotomy, a portion of pericardium was resected and the epicardium was abraded. Rabbits $(n=36)$ were randomly assigned to 4 treatment groups: control group, no bioresorbable polymeric film or expanded polytetrafluoroethylene; bioresorbable polymeric film group; expanded polytetrafluoroethylene group; and bioresorbable polymeric film + expanded polytetrafluoroethylene group. At 4 weeks post-sternotomy, pericardial adhesions were scored grossly for area and density of adhesions using an established 4-point (0-3) grading system.
\end{abstract}

Results: The bioresorbable polymeric film group had a significant reduction in mean adhesion score compared with the control group (control $=2.86 \pm 0.37$ vs bioresorbable polymeric film $=0.57 \pm 0.53, P<.0001$ ) and expanded polytetrafluoroethylene group (expanded polytetrafluoroethylene $=2.75 \pm 0.46$ vs bioresorbable polymeric film $=0.57 \pm 0.53, P<.0001)$. The bioresorbable polymeric film + expanded polytetrafluoroethylene group had a low adhesion profile similar to the bioresorbable polymeric film group (bioresorbable polymeric film + expanded polytetrafluoroethylene $=1.0 \pm 0$, vs bioresorbable polymeric film $=0.57 \pm 0.53$ ), but a considerably lower mean adhesion score than the expanded polytetrafluoroethylene group (expanded polytetrafluoroethylene $=2.75 \pm 0.46$, vs bioresorbable polymeric film + expanded polytetrafluoroethylene $=1.0 \pm 0, P<.0001$ )

Conclusions: Placement of bioresorbable polymeric film resulted in minimal pericardial adhesions compared with controls. The placement of bioresorbable polymeric film underneath expanded polytetrafluoroethylene at the time of sternal closure provides a novel combination to reduce the extent and severity of pericardial adhesions while providing a physical barrier between the sternum and the cardiac structures. (J Thorac Cardiovasc Surg 2011;141:789-95)

Cardiac reoperations are increasingly common, especially among pediatric patients with congenital cardiac disease, who frequently require reoperations or planned staged

\footnotetext{
From the Division of Cardiovascular-Thoracic Surgery, ${ }^{a}$ Children's Memorial Hospital; and the Department of Surgery, ${ }^{\mathrm{b}}$ Northwestern University Feinberg School of Medicine, Chicago, Ill.

Disclosures: This study was supported by SyntheMed, Inc, Iselin, New Jersey, which supported the funding of the project and provided the bioresorbable polymeric film. Dr Carl L. Backer is a consultant to SyntheMed, Inc. The authors had full control of the design of the study, methods, analysis of the data, and the written article.

Read at the 36th Annual Meeting of The Western Thoracic Surgical Association, Ojai, California, June 23-26, 2010.

Received for publication June 25, 2010; revisions received Oct 8, 2010; accepted for publication Nov 29, 2010.

Address for reprints: Sunjay Kaushal, MD, PhD, Children's Memorial Hospital, Division of Cardiovascular-Thoracic Surgery, 2300 Children's Plaza, mc 22, Chicago, IL 60614 (E-mail: skaushal@ childrensmemorial.org).

$0022-5223 / \$ 36.00$

Copyright (c) 2011 by The American Association for Thoracic Surgery

doi:10.1016/j.jtcvs.2010.11.043
}

repairs. Approximately $10 \%$ of current cardiac operations involve repeat sternotomies. ${ }^{1}$ Unfortunately, the repeat median sternotomy is a risk factor for reentry injury to the patient because of the presence of adhesions that obscure cardiac anatomy. ${ }^{2}$ Adverse events related to the reoperation lead to poor patient outcome and higher cost. ${ }^{3}$ These complications of reoperations have prompted experimental studies of how to prevent or decrease adhesion formation and thus decrease the risks associated with reoperation. Strategies for reducing reentry injury include insertion of a permanent barrier-like expanded polytetrafluoroethylene $(\mathrm{ePTFE})^{4}$ and, more recently, the use of bioresorbable films to reduce the extent and severity of adhesions. ${ }^{5,6}$ ePTFE can provide a permanent barrier beneath the sternum to shield the heart during future sternotomies. ${ }^{7}$ However, a strong inflammatory response underneath the ePTFE causes scarring and fixation of the membrane to areas of the epicardium, 


\section{Abbreviations and Acronyms \\ $\mathrm{BPF}=$ biodegradable polymeric film \\ ePTFE $=$ expanded polytetrafluoroethylene \\ $\mathrm{NTF}=$ neotissue fibrosis}

the control group, four 5-0 polypropylene sutures were placed at the edge of the pericardium. In the BPF group, BPF was placed over the epicardium and secured with four 5-0 sutures to the edge of the pericardium. In the ePTFE group, ePTFE was similarly placed on the epicardium. In the $\mathrm{BPF}+\mathrm{ePTFE}$ group, a combination of BPF underneath ePTFE was placed on the epicardium and secured to the pericardial edge (Figure 1). After treatment, a chest tube was temporarily inserted into the mediastinum and then removed on suction once the sternum and the remaining tissues were reapproximated.

which obscures the cardiac anatomy., ${ }^{2,7}$ The other alternative is biodegradable polymeric film (BPF), a composite of polyethylene glycol and polylactic acid, which has been shown to reduce the incidence and severity of retrosternal adhesions, but does not provide a permanent barrier because it is resorbed within 28 days. ${ }^{9,10}$

This study examines a novel combination of BPF and ePTFE to determine whether this combination has an advantage when compared with used alone by both decreasing adhesion formation and creating a permanent prosthetic barrier to protect from cardiac injury at sternal reentry. We used a standard experimental rabbit model ${ }^{5,9,11}$ and considered 4 treatment groups: (1) control, (2) BPF, (3) ePTFE, and (4) BPF + ePTFE. Each treatment group was evaluated 4 weeks after surgery to assess the extent and severity of adhesion formation by an independent observer who was unaware of the treatment groups. In addition, we performed blinded histology analysis that quantified the neotissue fibrosis (NTF) formation on the surface of the epicardium. ${ }^{12}$

\section{MATERIALS AND METHODS \\ Membranes}

BPF (REPEL-CV Adhesion Barrier; SyntheMed, Inc, Iselin, NJ), composed of polyethylene glycol and polylactic acid in a ratio of 1.5:1, was used in this study. The film was hydrated in sterile saline at room temperature for 15 minutes and then cut into a $5 \times 6$-cm oval shape for use during the surgery. ePTFE permanent prosthetic membrane (Gore PRECLUDE Pericardial Membrane; WL Gore \& Associates, Inc, Flagstaff, Ariz) was similarly cut and inserted.

\section{Animal Preparation}

The Children's Memorial Research Center Institutional Animal Care and Use Committee approved this protocol. Thirty-six New Zealand white female rabbits $(4 \mathrm{~kg})$ were randomly assigned to 1 of 4 groups: control $(n=9), \operatorname{BPF}(n=9)$, ePTFE $(n=9)$, and BPF+ePTFE $(n=9)$. Animals were tranquilized with acepromazine $(0.5 \mathrm{mg} / \mathrm{kg}$ subcutaneous $)$ and general anesthesia induced via ketamine $(40 \mathrm{mg} / \mathrm{kg})$ and xylazine $(7 \mathrm{mg} / \mathrm{kg})$, followed by immediate endotracheal intubation with a 3- or 4-mm tube. After initial induction, anesthesia was maintained intraoperatively with $2 \%$ isoflurane at $1 \mathrm{~L} / \mathrm{min}$ via inhalation. Intravenous access to the marginal ear vein was obtained, and animals were placed in a supine position for continuous cardiorespiratory monitoring throughout the procedure.

\section{Intraoperative Procedure}

The fur was removed from the chest, and the chest was prepped with Betadine. A midline sternotomy incision was performed, and an anterior pericardiotomy was performed from the left phrenic nerve to the right phrenic nerve. The epicardial surface was abraded for 5 minutes with a gauze square to produce punctuate bleeding and incite adhesion formation. In

\section{Macroscopic Adhesion Evaluation}

Four weeks after initial treatment, each rabbit was euthanized and underwent repeat sternotomy. Retrosternal adhesion formation was assessed visually at the apex, middle, and base of the central epicardial strip by an independent observer who was blinded to the treatment method. Adhesion density was qualitatively determined according to a standard adhesion scoring scale: $0=$ no adhesions; $1=$ mild adhesions, easy to dissect; $2=$ moderate adhesions; $3=$ severe cohesive adhesions, difficult to dissect. $^{10}$

\section{Microscopic Adhesion Evaluation}

Samples of right ventricular epicardial tissue were obtained and fixed in $10 \%$ phosphate-buffered formalin, and then embedded in paraffin and stained with hematoxylin-eosin for light microscopic evaluation to identify the extent of neofibrosis on the epicardial surface. We quantified the adhesion tissue thickness by determining the NTF thickness, composed of dense collagen and adipose tissue. ${ }^{12}$ By using Masson trichrome staining, the thickness of the NTF was analyzed by a blinded observer with the National Institutes of Health Image program (version 1.62; National Institutes of Health, Springfield, Va).

\section{Statistical Methods}

All data are presented as mean \pm standard deviation. Normally distributed data are compared by Student $t$ test to analyze variance.

\section{RESULTS}

The 36 rabbits had a sternotomy, were abraded for adhesion formation, and then were treated according to the assigned group: control, BPF, ePTFE, or BPF + ePTFE. One rabbit in the BPF group had a technical issue with displacement of the endotracheal tube during the operation, which led to death. Thus, 35 rabbits $(97.2 \%)$ survived the initial surgery with no postoperative complications.

\section{Gross Examination}

After 4 weeks, surviving rabbits $(n=35)$ were euthanized, a repeat sternotomy was performed, and gross examination was performed on the adhesions (Figure 2). In the control group, highly tenacious adhesions formed throughout the retrosternal region and dissection between the sternum and the epicardium was difficult, leading to injury to the myocardium in some rabbits. In the BPF group, visually there were fewer adhesions with a smooth epicardium that facilitated an easy dissection. Some rabbits in this group appeared to lack any adhesions, similar to the appearance at the initial sternotomy. In the PTFE group, the adhesions formed were nearly similar and dense as present in the control group. Finally, in the BPF+PTFE group, the adhesions 

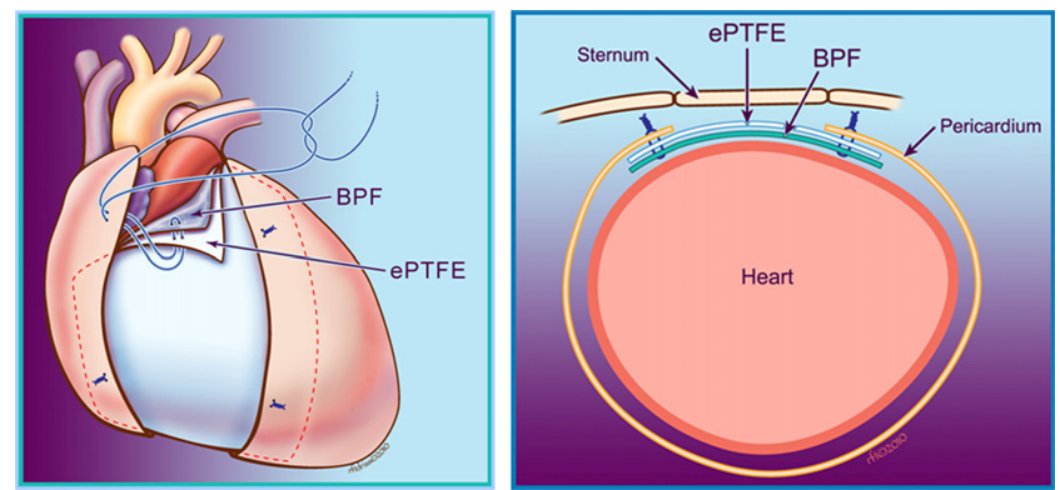

FIGURE 1. Technique of securing the BPF and ePTFE layers to the pericardial edge (frontal view, left; cross-section, right). BPF, Biodegradable polymeric film; $e P T F E$, expanded polytetrafluoroethylene.

were minimal and a smooth epicardium was present that was similar to the BPF group.

An independent observer who was unaware of the treatment quantified the adhesions by using a 4-point scoring system on 3 regions of the heart: apex, middle, and base (Figure 3). The mean adhesion score in the control group was $2.86 \pm 0.37$. In contrast, the BPF group had a score of $0.57 \pm 0.53$, and the BPF + ePTFE group had a similarly low adhesion score of $1.0 \pm 0$ with most of the adhesions above the ePTFE, against the sternum. The ePTFE group had a score of $2.75 \pm 0.46$, similar to the control group.
In addition, the ePTFE group had adhesions that were noted between the epicardium and the ePTFE membrane, as well as between the membrane and the sternum. None of the groups had adhesions located at the posterior or lateral portions of the heart. Furthermore, the regional distribution of the adhesions within the heart was uniform between the apex, middle, and base of the heart (Figure 4).

There was a significant reduction of adhesions between the $\mathrm{BPF}$ and the control groups (control $=2.86 \pm 0.37 \mathrm{vs}$ $\mathrm{BPF}=0.57 \pm 0.53, P<.0001)$ and compared with the ePTFE group $(\mathrm{ePTFE}=2.75 \pm 0.46$ vs $\mathrm{BPF}=0.57 \pm 0.53$,

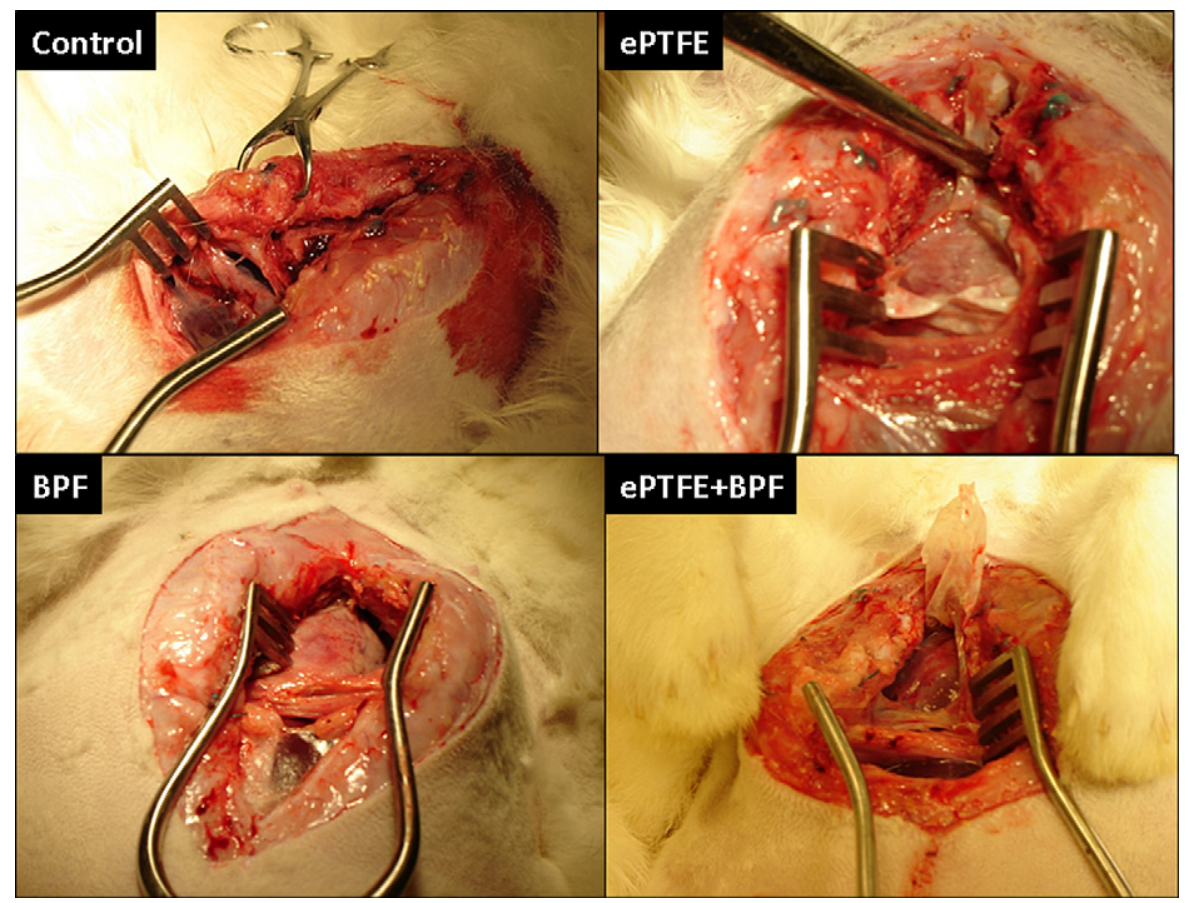

FIGURE 2. Operative photographs 4 weeks after initial surgery. The control group shows highly tenacious adhesions formed throughout the retrosternal region. The BPF group visually had fewer adhesions. In the ePTFE group, the adhesions formed were nearly similar and dense as in the control group. In the $\mathrm{BPF}+\mathrm{PTFE}$ group, the adhesions were minimal and a smooth epicardium was present that was similar to the BPF group. BPF, Biodegradable polymeric film; $e P T F E$, expanded polytetrafluoroethylene. 


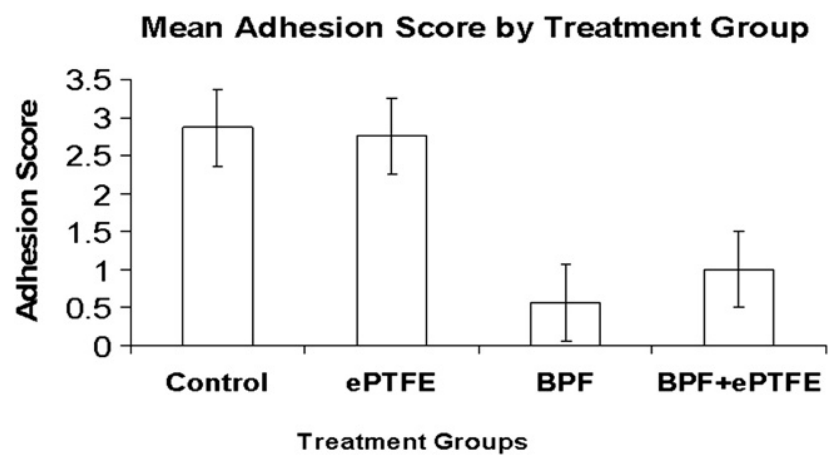

FIGURE 3. Bar graph showing mean adhesion score by treatment group. The mean adhesion score in the control group was $2.86 \pm 0.37$. In contrast, the BPF group had a score of $0.57 \pm 0.53$, and the BPF+ePTFE group had a similarly low adhesion score of $1.0 \pm 0$. The ePTFE group had a score of $2.75 \pm 0.46$, similar to the control group (control vs BPF, $P<.0001$; ePTFE vs BPF, $P<.0001$; ePTFE vs BPF + ePTFE, $P<.0001$; BPF + ePTFE vs $\mathrm{BPF}) . B P F$, Biodegradable polymeric film; $e P T F E$, expanded polytetrafluoroethylene.

$P<.0001)$.The BPF + ePTFE group had a low adhesion profile similar to the BPF group (BPF + ePTFE $=1.0 \pm 0$ vs $\mathrm{BPF}=0.57 \pm 0.53$ ), but considerably fewer adhesions than the ePTFE group $(\mathrm{BPF}+\mathrm{ePTFE}=1.0 \pm 0 \mathrm{vs}$ ePTFE $=2.75 \pm 0.46, P<.0001)$.

\section{Microscopic Analysis}

All specimens had an NTF layer composed of collagen and adipose tissue that formed above the epicardium (Figure 5). In the control group, a prominent, thick layer of NTF had formed that was consistent with the macroscopic findings (Figures 4 and 5). In both the BPF and $\mathrm{BPF}+\mathrm{ePTFE}$ groups, a thinner NTF layer was present compared with the control group. In the ePTFE group, a moderately thick layer of NTF was noted above the epicardium, similar to the control group. There was a significant difference in the mean adhesion tissue thickness among the different groups (Figure 6). The $\mathrm{BPF}+\mathrm{ePTFE}$ group showed significantly less tissue thickness than both the control and ePTFE groups (BPF+ePTFE vs control, $P<.001$; BPF + ePTFE vs ePTFE, $P<.001$ ), whereas there was no significant difference between the $\mathrm{BPF}$ and $\mathrm{BPF}+\mathrm{ePTFE}$ groups $(P=.08)$. The BPFtreated group had less neo-tissue thickness than the ePTFE group alone $(P<.001)$, which was a statistically significant difference.
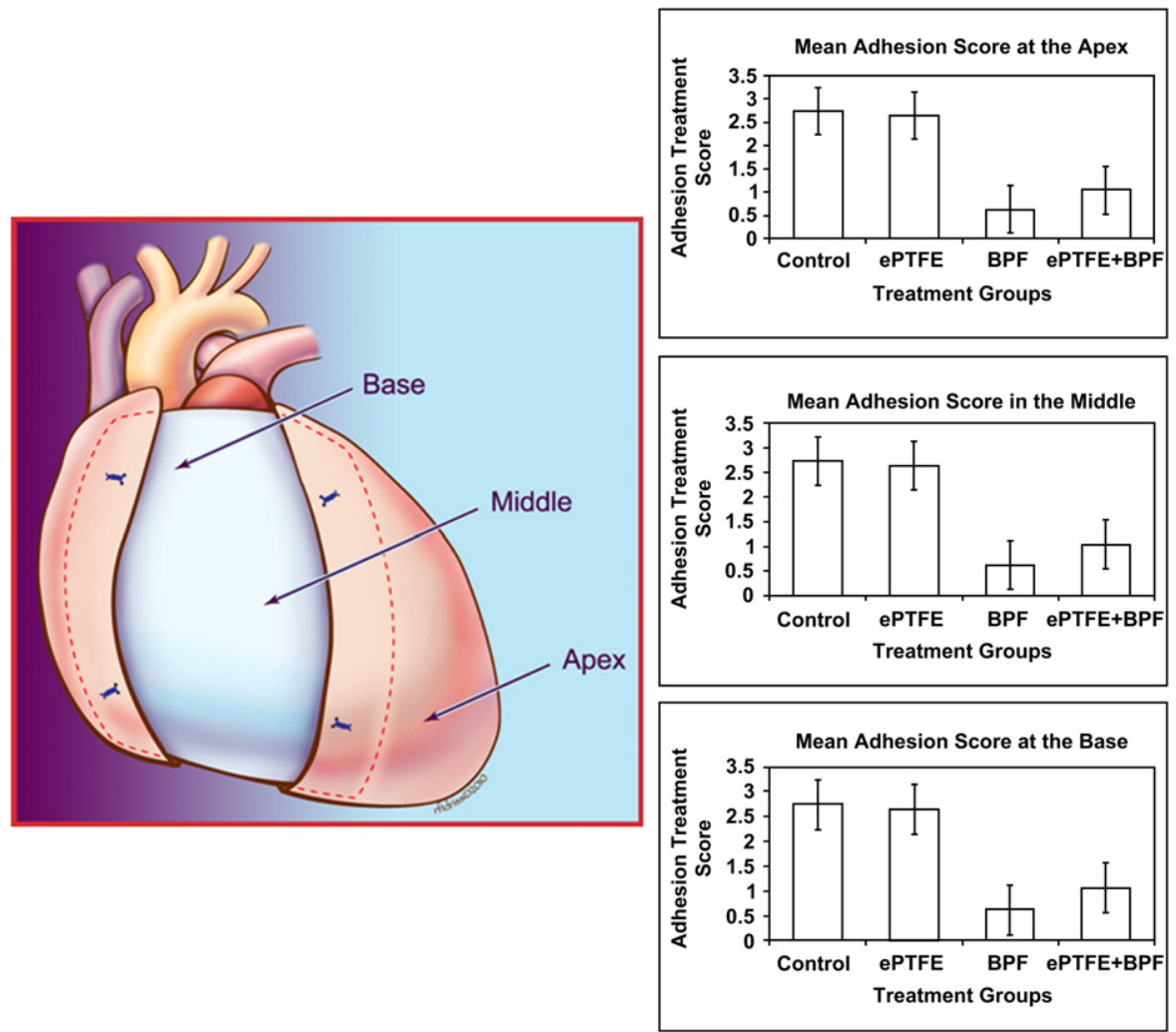

FIGURE 4. Regions of heart assessed by observer blinded to treatment group (left). Bar graphs (right) showing mean adhesion tenacity at the 3 regions of the heart for each treatment group. BPF and BPF+ePTFE groups had significantly lower adhesion tenacity at each of the 3 regions (base, middle, and apex of the heart) compared with the control and ePTFE only groups (control vs BPF, $P<.0001$; ePTFE vs BPF, $P<.0001$; ePTFE vs BPF + ePTFE, $P<.0001$; $\mathrm{BPF}+\mathrm{ePTFE}$ vs BPF). $B P F$, Biodegradable polymeric film; $e P T F E$, expanded polytetrafluoroethylene. $B P F$, Biodegradable polymeric film; $e P T F E$, expanded polytetrafluoroethylene. 

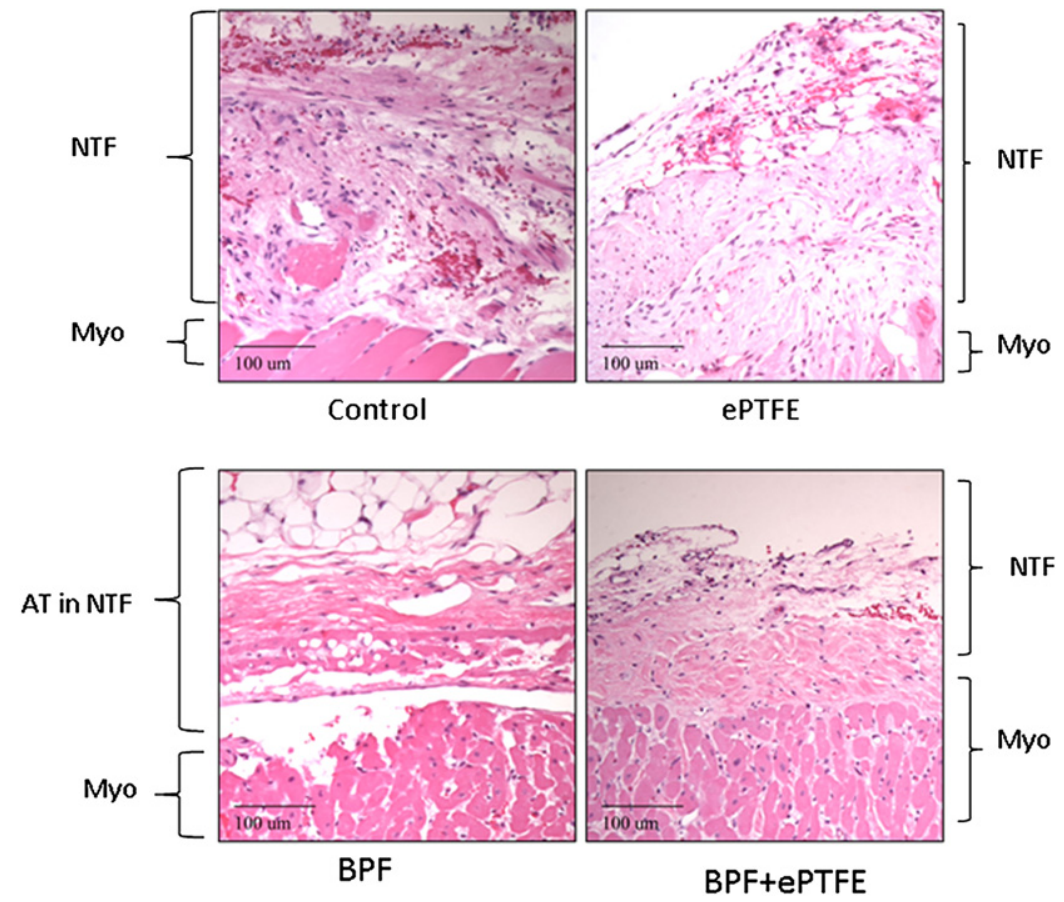

FIGURE 5. With the use of hematoxylin-eosin staining, the thickness of the NTF was analyzed from samples of right ventricular epicardial tissue. NTF layer was thinner in the BPF and BPF +ePTFE treatment groups compared with the control and ePTFE only groups. BPF, Biodegradable polymeric film; ePTFE, expanded polytetrafluoroethylene; $N T F$, neotissue fibrosis; $A T$, adipose tissue; $M y o$, myocardium.

\section{DISCUSSION}

This study demonstrated the efficacy of a bioresorbable polymeric film to reduce adhesion density between the epicardium and the sternum in a rabbit model. We further showed that the combination of BPF and ePTFE was equally effective in reducing adhesion density but also provided a physical barrier for safer sternal reentry. Many

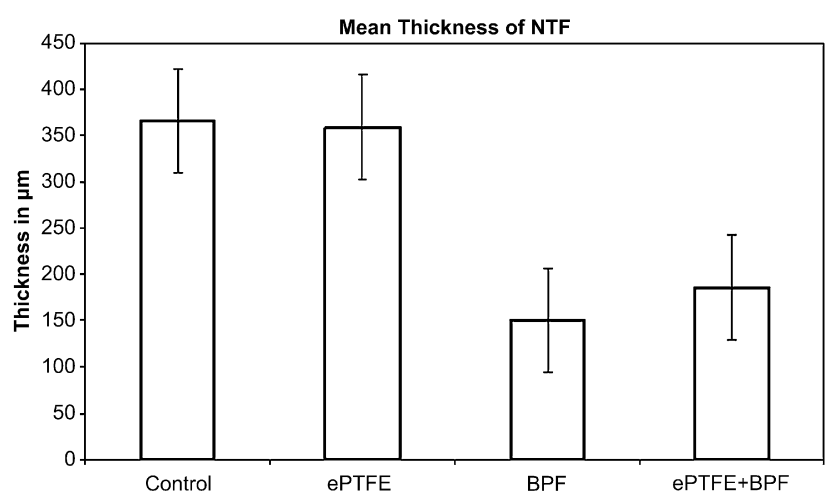

FIGURE 6. Bar graph showing mean adhesion thickness on the epicardial surface of the heart for each treatment group and control group. The $\mathrm{BPF}+\mathrm{ePTFE}$ group showed significantly less tissue thickness than both the control and ePTFE groups (BPF + ePTFE vs control, $P<.0001$; $\mathrm{BPF}+\mathrm{ePTFE}$ vs ePTFE, $P<.0001)$, whereas there was no significant difference between the BPF and BPF+ePTFE groups $(P=.08)$. A difference was seen between the BPF group and the PTFE group $(P<.0001)$. NTF, Neotissue fibrosis. congenital heart surgeons consider a physical barrier to be important in preventing injury to underlying cardiac structures, such as conduits and malposed vessels. With BPF placed underneath ePTFE, details of the epicardial cardiac anatomy were preserved and not obscured, which is typically seen when ePTFE alone is placed under the sternum. The combination of BPF and ePTFE elicits this effect by decreasing adhesion formation under the ePTFE, resulting in a superior benefit than when either is used separately. ePTFE provides a physical barrier, and BPF reduces the extent and severity of adhesions. These results have important clinical implications for patients who are to undergo repeat sternotomies.

Previous studies have determined that the optimal polymer ratio for BPF is 1.5:1 polyethylene glycol to polylactic acid. ${ }^{5,9}$ This maximizes the initial mechanical strength and flexibility but allows for complete resorption by 28 days post-implant. A recent prospective randomized trial of BPF in patients with congenital cardiac disease demonstrated safety and efficacy in reducing the extent and severity of postoperative adhesion formation. ${ }^{10}$ This material is now approved by the Food and Drug Administration for patients aged 21 years or less who expect to undergo a repeat sternotomy. Despite this success with BPF, a limitation is that the film is resorbed, leaving no physical barrier between the sternum and the epicardium. The combination of BPF and ePTFE circumvents this limitation by decreasing adhesion formation and providing a barrier for sternal reentry. 
Patients with congenital cardiac disease are now living longer after their initial or palliative operations, which correlates with more repeat sternotomies for reoperations or transplantation. ${ }^{2}$ The difficulty of repeat sternotomies lies with adhesion formation between the sternum and the epicardium that obscures the anatomy and thus predisposes to vascular injury, myocardial lacerations, and increased morbidity. ${ }^{3}$ The genesis of mediastinal adhesions is a multistep process that unfolds over a period of 2 to 3 weeks. The presence of blood and serosal injury initiates the formation of adhesions in the mediastinum. Initially, an exudative phase exists with fluid, inflammatory cells, and fibrin deposition. This is followed by a desquamation phase, with injury to the mesothelial cells and aggregation of inflammatory cells adhering to mesothelial cells along with further fibrin deposition. The third phase is collagen deposition and blood vessel growth in the newly formed connective tissue. The last phase is adhesion formation. BPF decreases adhesions by providing a temporary barrier to fibrin bridges connecting apposing surfaces during the critical first several weeks after the sternotomy. ${ }^{10}$

Several strategies have been proposed to reduce adhesion formation: xenograft membranes, prosthetic membranes, fibrolytic solutions, and resorbable membranes. ${ }^{13}$ Xenogenic materials have shown evidence of infection and late calcification resulting in limited clinical use. ${ }^{14}$ Synthetic nonresorbable materials include ePTFE, silicone rubber, polyethylene film, and Dacron mesh. ${ }^{4-7,15-17}$ The most common synthetic material used in the clinical setting is ePTFE, but this has a major limitation because of a vigorous inflammatory reaction that creates a fibrous capsule over the surface of the heart. ${ }^{7,17}$ Other methods used to inhibit pericardial adhesions have included fibrolytic agents that alter the balance between fibrin deposition toward fibrin dissolution. ${ }^{11}$ A limitation clinically for these fibrolytic solutions are the side effects of bleeding and altered healing ability. Bioresorbable materials have an advantage based on their ability to prevent adhesion formation with no foreign body response like that seen with ePTFE. Hyaluronic acid biocompatible membrane (Seprafilm, Genzyme, Cambridge, MA) was shown to facilitate reoperations in complex congenital cardiac surgery, but this study was not blinded or randomized. ${ }^{18}$ Naito and colleagues, ${ }^{12}$ from Tokyo Women's University, performed a similar study to ours but with Seprafilm (a hyaluronic acid bioabsorbable membrane). They concluded that the combined use of Seprafilm with either PTFE or autologous pericardium effectively reduced the formation of pericardial adhesions beneath the physical barrier. Their conclusions are similar to ours with a different bioresorbable polymeric film that does not have Food and Drug Administration approval for mediastinal applications.

\section{Limitations}

Our study has several limitations. There are intrinsic differences between the rabbit model and humans that will not be solved until a human clinical trial is conducted. Another limitation is that other contributing factors for adhesion formation were lacking in this rabbit model (ie, cardiopulmonary bypass). Finally, the duration of the study was 28 days, which is far less than the shortest reoperation period of 3 to 6 months commonly observed in patients with congenital cardiac disease undergoing single ventricle palliation operations.

\section{CONCLUSIONS}

Bioresorbable polymeric film in combination with ePTFE reduced the extent and severity of adhesion formation between the heart and the ePTFE and provided a physical barrier during sternal reentry. With this novel combination, ePTFE did not induce a capsule formation over the heart, which typically obscures the anatomy of the heart. These data suggest that the combination therapy of BPF and ePTFE provides a unique method for preventing adverse outcomes in cardiac reoperations and making sternal reentry safer. A human clinical trial is needed to validate this experimental model.

The authors thank Dr Jose Hernandez, DVM, for assistance during the operations and care of the animals.

\section{References}

1. Follis FM, Pett SB Jr, Miller KB, Wong RS, Temes RT, Wernly JA. Catastrophic hemorrhage on sternal reentry: still a dreaded complication? Ann Thorac Surg. 1999;68:2215-9.

2. Kirshbom PM, Myung RJ, Simsic JM, Kramer ZB, Leong T, Kogon BE, et al. One thousand repeat sternotomies for congenital cardiac surgery: risk factors for reentry injury. Ann Thorac Surg. 2009;88:158-61.

3. Roselli EE, Pettersson GB, Blackstone EH, Brizzio ME, Houghtaling PL, Hauck R, et al. Adverse events during reoperative cardiac surgery: frequency, characterization, and rescue. J Thorac Cardiovasc Surg. 2008;135:316-23.

4. Loebe M, Alexi-Meskhishvilli V, Weng Y, Hausdorf G, Hetzer R. Use of polytetrafluoroethylene surgical membrane as a pericardial substitute in the correction of congenital heart defects. Tex Heart Inst J. 1993;20:213-7.

5. Okuyama N, Rodgers KE, Wang CY, Girgis W, Oz M, St Amand K, et al. Prevention of retrosternal adhesion formation in a rabbit model using bioresorbable films of polyethylene glycol and polylactic acid. J Surg Res. 1998;78:118-22.

6. Mitchell JD, Lee R, Neya K, Vlahakes GJ. Reduction in experimental pericardial adhesions using a hyaluronic acid bioabsorbable membrane. Eur J Cardiothorac Surg. 1994;8:149-52.

7. Jacobs JP, Iyers RS, Weston JS, Amato JJ, Elliott MJ, de Leval MR, et al. Expanded PTFE membrane to prevent cardiac injury during resternotomy for congenital heart disease. Ann Thorac Surg. 1996;62:1778-82.

8. Meus PJ, Wernly JA, Campbell CD, Takanashi Y, Pick RL, Zhao-Kun Q, et al. Longterm evaluation of pericardial substitutes. J Thorac Cardiovasc Surg. 1983;85:54-8.

9. Okuyama N, Wang CY, Rose EA, Rodgers KE, Pines E, diZerega GS, et al. Reduction of retrosternal and pericardial adhesions with rapidly resorbable polymer films. Ann Thorac Surg. 1999;68:913-8.

10. Lodge AJ, Wells WJ, Backer CL, O’Brien JE Jr, Austin EH, Bacha EA, et al. A novel bioresorbable film reduces postoperative adhesions after infant cardiac surgery. Ann Thorac Surg. 2008;86:614-21.

11. Wiseman DM, Kamp L, Linsky CB, Jochen RF, Pang RH, Scholz PM. Fibrinolytic drugs prevent pericardial adhesions in the rabbit. J Surg Res. 1992;53:362-8. 
12. Naito Y, Shin'oka T, Hibino N, Matsumura G, Kurosawa H. A novel method to reduce pericardial adhesion: a combination technique with hyaluronic acid biocompatible membrane. J Thorac Cardiovasc Surg. 2008;135:850-6.

13. Tsukihara H, Takamoto S, Kitahori K, Matsuda K, Murakami A, Novick RJ, et al. Prevention of postoperative pericardial adhesions with a novel regenerative collagen sheet. Ann Thorac Surg. 2006;81:650-7.

14. Eng J, Ravichandran PS, Abbott CR, Kay PH, Murday AJ, Shreiti I. Reoperation after pericardial closure with bovine pericardium. Ann Thorac Surg. 1989;48: 813-5.

15. Laks H, Hammond G, Geha AS. Use of silicone rubber as a pericardial substitute to facilitate reoperation in cardiac surgery. J Thorac Cardiovasc Surg. 1981;82: $88-92$.

16. Revuelta JM, Garcia-Rinaldi R, Val F, Crego R, Duran CM. Expanded polytetrafluoroethylene surgical membrane for pericardial closure. An experimental study. J Thorac Cardiovasc Surg. 1985;89:451-5.

17. Bunton RW, Xabregas AA, Miller AP. Pericardial closure after cardiac operations. An animal study to assess currently available materials with particular reference to their suitability for use after coronary artery bypass grafting. J Thorac Cardiovasc Surg. 1990;100:99-107.

18. Walther T, Rastan A, Dähnert I, Falk V, Jacobs S, Mohr FW, et al. A novel adhesion barrier facilitates reoperations in complex congenital cardiac surgery. J Thorac Cardiovasc Surg. 2005;129:359-63.

\section{Discussion}

Dr Gordon Cohen (Seattle, Wash). Because both the PTFE and the BPF are commercially available, many surgeons have experienced using one or the other or maybe even both, and not surprisingly have an opinion as to why one is better than another. However, I have yet to hear of this novel approach of anyone using them both together. As is common in our specialty, we often just start doing things because we think they are better, but you designed a straightforward and practical study to try and actually answer this question.

I have a few questions for you.

1. Out of curiosity, how do you blind the evaluator to the presence or absence or PTFE?

2. Is 4 weeks a long enough period for significant adhesions to form? Is there some relationship between the different materials and time, that is, would it not have been better to evaluate the adhesions at different time points (eg, 1, 3, and 6 months) to really understand the differences?

3. You must have tried this combination in patients. What has been your experience in actual clinical practice?

4. Finally, just an opinion, do you think that some sort of PTFE and BPF combination should be a standard of care for patients who will certainly undergo repeat cardiac surgery? Because it decreases the risk associated with reoperation and potentially decreases time, which we could speculate translates to a reduction in costs.

Dr Kaushal. The first question relates to the blinded observer. I might have mentioned that in the text, but I have changed that subsequently because of course you cannot have a blinded observer to PTFE. The observer was actually independent and not aware of each treatment group, so that is why we used that as an observer. It was not blinded.

The second question asks why did I look at 4 weeks and was there a long-lasting effect of preventing adhesions beyond the 1 month, to 3 or 6 months. The reason why I chose 4 weeks is that we have additional data that we did not present where we have looked at the adhesion formation along an interval course, spanning 1,3, and 6 months, and what we noticed was that there was no difference of adhesion formation in that time period, so that is why we subsequently reduced our evaluation to just 1 month.

Your third question involves the clinical utility of this and have we used that in our patients. We have not used that currently. Our plans are to start a clinical trial this fall. We think that using this combination will prevent adhesion formation. I think the best way to analyze something before it is the standard of care, which deals with your fourth question, is that we have to rigorously study this and make sure that it is safe and effective.

Speaker. If the physical barrier and BPF work so well, have you ever tried using a sandwich with BPF on one side and the other side underneath the sternum so it prevents the adhesions and the PTFE is in the middle?

Dr Kaushal. That is a very good thought, and in fact our next set of experiments are exactly that. As I mentioned in our PTFE group where we used the combination, there was adhesion formation between the sternum and the PTFE, so our thoughts were, as you just mentioned, that maybe we can use a triple sandwich where we use BPF, PTFE, and BPF to prevent adhesion formation throughout that area.

Dr Pirooz Eghtesady (Cincinnati, Ohio). Two questions. What is the cost for these 2 things, and, at least from my perspective, the PTFE does a nice job ensuring safety during reentry, but a big pain is when you have to dissect the right atrium off the pericardium, dissect between the aorta and the pulmonary more down below. Does this stuff sort of trickle down from the surface and prevent those adhesions?

I do not know if one could do this. I think a corollary would be if you want to make it clinical conversion either looking at the time it takes to have a certain exposure if you did an animal study or injury during that process as you were trying to set things up.

Dr Kaushal. I do not have the exact price for the BPF, but I can get that for you if you require it.

Your second question deals with the healing properties of this and whether you can apply it to different areas, especially the right atrium. The healing quality of this is actually very good. The compliance of this film is good in the sense that once you soak it for 5 minutes in saline it is pliable, and you can actually cut it to the shape or size that you require and apply it to the areas you need, so if you wanted to have more on the right atrium you can actually do that. I think that is easy. We did not do that in this study.

The timing was actually looked at in the original study performed with the infant surgeries in those patients, and there was a decrease in time to perform the dissection of adhesion formation. So that has been looked at in the clinical trial, but it is a good point. We will look at that in the next trial we perform. I think as we have gained experience with re-do sternotomies, our mortality rate has definitely decreased, and there have been 2 different reports stating the ease of a re-do, is that a risk factor for mortality, and it is not really a risk factor anymore for mortality, but I think what will come out of that is why we are pursuing this. This will be another adjunct in preventing injuries that do not result in mortality but in morbidity. 